Research Paper

\title{
Extracorporeal Shockwave Therapy Enhances Expression of Pdia-3 Which Is a Key Factor of the 1 $\alpha, 25$-Dihydroxyvitamin D 3 Rapid Membrane Signaling Pathway in Treatment of Early Osteoarthritis of the Knee
}

\author{
Shan-Ling Hsu ${ }^{1,2}$, Jai-Hong Cheng ${ }^{1,3 凶}$, Ching-Jen Wang ${ }^{1,2}$, Jih-Yang Ko ${ }^{1,2}$, Chih-Hsiang Hsu ${ }^{2}$ \\ 1. Center for Shockwave Medicine and Tissue Engineering, \\ 2. Department of Orthopedic Surgery, \\ 3. Medical Research, Kaohsiung Chang Gung Memorial Hospital and Chang Gung University College of Medicine, Kaohsiung, Taiwan.
}

$\square$ Corresponding authors: Shan-Ling Hsu, Department of Orthopedic Surgery, Kaohsiung Chang Gung Memorial Hospital, 123 Tai-Pei Road, Niao Sung District, Kaohsiung, Taiwan 833. Email: hsishanlin@yahoo.com.tw and Jai-Hong Cheng, Center for Shockwave Medicine and Tissue Engineering, Kaohsiung Chang Gung Memorial Hospital, 123 Tai-Pei Road, Niao Sung District, Kaohsiung, Taiwan 833. Email: cjh1106@cgmh.org.tw

(1) Ivyspring International Publisher. This is an open access article distributed under the terms of the Creative Commons Attribution (CC BY-NC) license (https://creativecommons.org/licenses/by-nc/4.0/). See http://ivyspring.com/terms for full terms and conditions.

Received: 2017.03.28; Accepted: 2017.07.05; Published: 2017.09.19

\begin{abstract}
The goal of our research was demonstrated that multiple molecules in microenvironments of the early osteoarthritis (OA) joint tissue may be actively responded to extracorporeal shockwave therapy (ESWT) treatment, which potentially regulated biological function of chondrocytes and synovial cells in early OA knee. We demonstrated that shockwave treatment induced the expression of protein-disulfide isomerase-associated 3 (Pdia-3) which was a significant mediator of the 1a,25-Dihydroxyvitamin $\mathrm{D} 3(1 \alpha, 25(\mathrm{OH}) 2 \mathrm{D} 3)$ rapid signaling pathway, using two-dimensional electrophoresis, histological analysis and quantitative polymerase chain reaction (qPCR). We observed that the expression of Pdia-3 at 2 weeks was significantly higher than that of other group at 4,8 , and 12 weeks post-shockwave treatment in early OA rat knee model. The other factors of the rapid membrane signaling pathway, including extracellular signal-regulated protein kinases 1 (ERK1), osteopontin (OPG), alkaline phosphatase (ALP), and matrix metallopeptidase 13 (MMP13) were examined and were found to be significantly increased at 2 weeks post-shockwave treatment by $\mathrm{qPCR}$ in early OA of the knee. Our proteomic data revealed significant Pdia-3 expression in microenvironments of OA joint tissue that could be actively responded to ESWT, which may potentially regulate the biological functions of chondrocytes and osteoblasts in the treatment of the early OA of the knee.
\end{abstract}

Key words: protein-disulfide isomerase-associated 3, osteoarthritis, extracorporeal shockwave therapy, 1a,25-Dihydroxyvitamin D3 signaling pathway, two dimensional electrophoresis

\section{Introduction}

Osteoarthritis (OA), one of the most common causes of musculoskeletal disorders in the developed countries [1], is characterized by cartilage attrition, reduced subchondral bone remolding, osteophyte formation and synovial inflammation, and factors inducing cartilage degeneration such as an inappropriate mechanical load [2], disturbed biochemical regulation [2] and genetic mutation [3] are potential etiologic causes of OA.

Osteoarthritis had been considered to be primarily a cartilage disorder characterized by cartilage degradation. Intensive inflammatory 
cytokines such as interleukin, tumor necrosis factor and proteases secreted from joint component cells caused by abnormal mechanical force are mainly responsible for acceleration of cartilage damage, loss of compensatory synthesis and eventually deterioration of the function of extra-cellular matrix organization [4-6]. Dysregulation of cartilage homeostasis caused by intensive chondrocyte apoptosis has been reported to be a potent pathological activity in the development of OA. Disturbance of oxidative stress [7], proapoptotic/antiapoptotic regulation [8, 9] and mitochondrial dysfunction [10] have been proposed to modulate chondrocyte survival in the progression of $\mathrm{OA}$; however, the molecular mechanism by which chondrocytes propagate towards programmed cell death has not been clearly defined.

The 1a,25-Dihydroxyvitamin D3 (1a,25(OH) 2D3) is essential in calcium homeostasis for the regulation of endochondral ossification [11]. In vitamin $\mathrm{D}$ deficiency, bone matrix synthesis and cartilage growth are inhibited. Vitamin D has been investigated to action on osteoblasts and growth plate chondrocytes through classic nuclear vitamin D receptor (VDR) and Pdia-3. Pdia-3 has been demonstrated to be a key factor in 1a,25(OH)2D3-induced phospholipase A2 (PLA2) and protein kinase $\mathrm{C}$ (PKC) activation in addition to downstream responses to gene transcription $[12,13]$.

A growing body of evidence has demonstrated that ESWT promotes tissue repair in various tissues and initiates biological responses [14, 15]. It has been reported that ESWT ameliorated experimental osteoarthritic cartilage damage and altered the pattern of angiogenesis [15]. The preliminary proteomic data revealed significant proteins in abundance that warranted further characterization [16-18]. These proteins of interest have been reported to participate in the cellular response to physical stress, calcium homeostasis, chemotaxis and lipid oxidative stress in several tissue types in pathological contexts. Therefore, we hypothesized that multiple molecules in joint tissue microenvironments may be actively responded to ESWT treatment, which potentially regulates the survival and biological function of chondrocytes in OA of the knee. To test the null hypotheses, we conducted studies to delineate the active responsive molecules in ESWT-regulated biological responses using a comparative proteomic technique which assisted with the construction of the molecular mechanism of alleviation of OA, and clarified whether ESWT changed the molecular signaling to interfering joint microstructures. Based on these translational experimental data, we were able to further explore a new treatment regime with good potential for rescuing joint injury in $\mathrm{OA}$ of the knee.

\section{Materials and Methods}

\section{Study Design}

The Institutional Review Board approved the animal experiment procedure followed in this study. All studies were performed in accordance with the guidelines for the care of animals used in experiments. All procedures and protocols were approved by the Institutional Animal Care and Use Committee of Chang Gung Memorial Hospital, Taiwan. One-hundred forty-four male four-month-old Sprague-Dawley rats with a body weight ranging from 250 to $275 \mathrm{~g}$ were used in this study. The rats were randomly divided into three groups, with 48 rats in each group, and 12 rats for each time course ( $n=48$ per group). The rats in the normal control (NC) group received neither the anterior cruciate ligament transection (ACLT) nor ESWT, and served as the baseline control. The rats in the OA group underwent ACLT, but received no ESWT. The rats in the OA+ESWT group underwent ACLT and received ESWT (800 impulses at 0.18 $\mathrm{mJ} / \mathrm{mm}^{2}$, the frequency was set at 4) to the subchondral bone of the medial tibia condyle. The 12 rats in each group were sacrificed at 2, 4, 8 and 12 week(s) post-surgery. Of the 12 rats at 2 week(s) post-surgery, the articular cartilage and subchondral bone of tibia of six rats were used for proteome analysis and the joints of another six rats for immunohistochemistry analysis.

\section{Experimental model of OA of the knee}

Four-month-old male Sprague-Dawley rats were anesthetized using intraperitoneal injection of pentobarbital ( $50 \mathrm{mg} / \mathrm{Kg}$ body weight). The left knees of the animals underwent surgery comprising medial parapatellar arthrotomy and ACLT to induce ACLT-mediated OA of the knee, as previously described [19]. Animals were allowed unrestricted weight-bearing and activity as tolerated. The left knee was excised after animals were sacrificed according to each time course.

\section{Histological analysis}

Whole knee joints were fixed in $4 \%$ phosphate-buffered paraformaldehyde, decalcified in $10 \%$ phosphate-buffered EDTA, then embedded in paraffin. Specimens were longitudinally cut into $4 \mu \mathrm{m}$ sections for hematoxylin and eosin staining (Sigma-Aldrich, USA). Histomorphometry of the articular cartilage was evaluated using the 14 point Mankin scoring system (for structural integrity, from $0=$ normal to $6=$ complete disorganization; for cells, 
from $0=$ normal to 3 = hypocellularity; for cartilage height, from $0=$ normal to $4=$ complete loss; for tidemark integrity, from $0=$ normal to $1=$ disruption). Twelve sections from 6 rats were measured under 100 $x$ magnification using a Zeiss Axioskop 2 plus microscope (Carl Zeiss Microimaging, Germany) with a cool CCD camera and Image-Pro Plus image analysis software (SNAP-Pro c.f. Digital kit; Media Cybernetics, USA). In some cases, the specimens were fixed in absolute alcohol, embedded in glycolmethylacrylate (Sigma-Aldrich, USA), then cut longitudinally into $8 \mu \mathrm{m}$ sections using a rotary tungsten steel-bladed microtome for von Kossa's and tartrate-resistant acid phosphatase histochemical staining (Sigma-Aldrich, USA). All sections were independently assessed by two individuals. Cartilage destruction was assessed using the Mankin scoring system [20]. Each section was evaluated using the Mankin histological and histochemical grading system, including structural changes in all layers of the uncalcified cartilage, tidemark integrity and hematoxylin and eosin staining.

\section{Two-dimensional electrophoresis in early OA knee and ESWT treatment}

The articular cartilage and subchondral bone of tibia of six rats were mixed and used for proteome analysis at two weeks in sham, OA and OA+ESWT groups. Samples of $250 \mu \mathrm{g}$ of protein from two comparative subjects were first applied to immobilize $\mathrm{pH}$ 3-10 nonlinear gradient strips with isoelectric focusing by using an Ettan ${ }^{\mathrm{TM}}$ IPGphor II/3system. Proteins in the strips were separated by $15 \%$ SDS-PAGE and silver-stained using PlusOne Silver Staining Kit (GE Healthcare, USA) according to the manufacturer's instructions. Protein spots in gels were scanned using an Amersham Image Scanner. Image analysis, spot-matching and spot intensity were performed and analyzed using Bio-Rad Proteoweaver 2 D Analysis Software Version 4.0 (Radius limit: 4; intensity limit: 2000; contrast limit: 50; border contrast: 0.2 ; active spots intensity warning limit: 5000) [21].

\section{MALDI-TOF/mass spectrometry (MS) and liquid chromatography/MS}

Spots of interest were excised and washed with $10 \mathrm{mM}$ ammonium bicarbonate and $50 \%$ acetonitrile in $10 \mathrm{mM}$ ammonium bicarbonate. After washing with deionized water and destaining with acetonitrile, the dried gels were digested by trypsin at $30^{\circ} \mathrm{C}$ for 4 h. The trypsin digest was extracted by trifluoroacetate, and aliquots of the digest were loaded onto an AnchorChip for one peptide mass fingerprinting (PMF) from matrix-assisted laser desorption/ time of flight (MALDI-TOF), and TOF/TOF MS/MS analysis of fragment peptides was performed using FlexControlTM software. Peptide mass data were submitted to the NCBI and the Swiss-Port database using MASCOT (with significance at $\mathrm{P}$-value $<0.05$ at scores over 30 ) search engines for peptide matching.

\section{Quantitative RT-PCR}

The primers of bone and cartilage biomarkers including OPG, ALP, MMP13, collagen type II and aggrecan were detected by quantitative RT-PCR (Table 1). Total RNA was extracted and purified from knee joint tissue using QIAzol reagent. Total RNA (1 $\mu \mathrm{g}$ ) was reverse transcribed into cDNA. The $25 \mu \mathrm{L}$ of PCR mixture containing cDNA template equivalent to $20 \mathrm{ng}$ total RNA, $2.5 \mu \mathrm{M}$ of each forward, and reverse primer and $2 \mathrm{X}$ iQTM SYBR green supermix was amplified using the $\mathrm{iCycler} \mathrm{iQ}{ }^{\circledR}$ Real time $\mathrm{PCR}$ detection system with an initial melt at $95^{\circ} \mathrm{C}$ for $5 \mathrm{~min}$ followed by 40 cycles at $94{ }^{\circ} \mathrm{C}$ for $15 \mathrm{sec}, 52{ }^{\circ} \mathrm{C}$ for 20 sec and $72{ }^{\circ} \mathrm{C}$ for $30 \mathrm{sec}$ using the following primer oligonucleotide sequences followed by PCR amplification using responsive molecules and rat $18 \mathrm{~S}$ rRNA primers (forward: 5'-GCAGCTAGGAATAATGGAATAGGA-3'; reverse: 5'-TAATGAAAACATTCTTGGCAAATG-3'). The number of amplification steps required to reach an arbitrary intensity threshold $(\mathrm{Ct})$ was computed. The relative gene expression level was presented as $2(-\Delta \mathrm{Ct})$, where $\Delta \mathrm{Ct}=\mathrm{Ct}$ target-Ct $18 \mathrm{~S}$ rRNA. The Fold change for the treatment was defined as the relative expression as compared with the vehicle and was calculated as 2- $\Delta \Delta \mathrm{Ct}$, where $\Delta \Delta \mathrm{Ct}=\Delta \mathrm{Ct}$ treatment $-\Delta \mathrm{Ct}$ vehicle.

Table 1. The primers were used for qPCR in this study.

\begin{tabular}{llll}
\hline Primer name & Type & Length & Sequence $\left(5^{\prime}-3^{\prime}\right)$ \\
\hline Rat Pdia-3 & Forward & 20-mer & GAGGCTTGCCCCTGAGTATG \\
Rat Pdia-3 & Reverse & 19-mer & GTTGGCAGTGCAATCCACC \\
ERK1 & Forward & $20-\mathrm{mer}$ & AGCTGCTAAAGAGCCAGCAG \\
ERK1 & Reverse & $20-\mathrm{mer}$ & GCAAGGCCAAAATCACAGAT \\
$\begin{array}{l}\text { Osteopontin } \\
\text { Osteopontin }\end{array}$ & Forward & 20-mer & GTTCTTGCACAGCTTCACCA \\
$\begin{array}{l}\text { Alkaline } \\
\text { phosphatase }\end{array}$ & Forward & $20-\mathrm{mer}$ & AAACAGCCCAGTGACCATTC \\
$\begin{array}{l}\text { Alkaline } \\
\text { phosphatase }\end{array}$ & Reverse & 20-mer & GGGAAGAAGCCCTTCACAGC \\
$\begin{array}{l}\text { MMP13 } \\
\text { MMP13 }\end{array}$ & Forward & 20-mer & GAGGTGAAAAGAGTTCTGCTCA \\
\hline
\end{tabular}

\section{Immunohistochemistry}

Sections were hybridized with relative antibodies against candidate proteins and analyzed using a streptavidin conjugated horseradish peroxidase (HRP) detection system (BioGenex, USA). Immunoreactivity of specimens was demonstrated using a HRP-3'-, 3'diaminobenzidine cell and tissue 
staining kit (R\&D systems, USA) according to the manufacturer's instructions. Pdia-3 antibody (ab13506, mouse monoclonal at 1:50 dilution; Abcam, USA) against discovered molecules were used. Sections were then incubated with rabbit anti-mouse biotinylated secondary antibody with streptavidin conjugated to HRP, followed by chromogen solution and counterstaining with hematoxylin. Sections were finally dehydrated and mounted. Sections without primary antibodies were employed as negative controls for immunostaining. The numbers of positive immunolabeled cells and total cells in each area were counted from five random areas in three sections of the same specimen, and the percentages of positive-labeled cells were calculated. All images were captured by using a cool CCD camera (Media Cybernaetics, USA). Images were analyzed by manual counting and confirmed by using an image-pro Plus Image-analysis software (Media Cybernetics, USA).

\section{Statistical analysis}

All values were expressed as the mean \pm standard error. One-way ANOVA and Tukey tests were used to assess the differences among the groups. The level of statistical significance was set at $\mathrm{P}<0.05$.

\section{Results}

\section{The effect of shockwave therapy on articular cartilage}

In macroscopically-normal articular cartilage, some changes in the cell distribution of the superficial layer are observed. Furthermore, the differences in knees suffering from OA before and after shockwave treatment are pronounced. Application of ESWT to the subchondral bone of the medial tibia condyle resulted in regression of $\mathrm{OA}$ in the knees of rats. In the OA+ESWT group, the articular cartilage, with a Mankin score ranging from 2 to 5 , was better preserved than in the OA group, with a Mankin score ranging from 4 to 8 , from 2 to 12 week(s) post-treatment (Fig. 1A and 1B). In the OA group, significantly greater articular cartilage degeneration was evident, as shown by the wide Mankin score range.

\section{The index surgery of OA knee and two-dimensional electrophoresis analysis}

The articular cartilage and subchondral bone of tibia of left knee were mixed and used for proteome analysis with conducted by two-dimensional gel electrophoresis and mass spectrometry. Protein spots in gel were developed by silver staining and scanned using an Amersham ImageScanner. The level of significance of the spots was analysis with a 1.5-fold increase or decrease in intensity by using an
Amersham ImageScanner analysis. After image scanning, twelve spots were chosen and found to be differentially abundant, including 9 spots with a decreased intensity and 3 with an increased signal, in the OA+ESWT group as compared with the normal control (NC) group and the OA group at 2 week(s) after index surgery (Fig. 2).

The levels of the 8 identified proteins differed significantly by Mascot score and NCBI BLAST score between the shockwave-treated OA group and the OA group, and also changed with disease development (Fig. 3). Among these proteins, two were up-regulated (Pdia-3 and guanine nucleotide-binding protein subunit beta-2-like). Moreover, six proteins were identified as being suppressed in the OA+ESWT group (Beta-enolase, chloride intracellular channel protein 1, malate dehydrogenase, purine nucleoside phosphorylase, creatine kinase M-type and L-lactate dehydrogenase A chain) (Table 2). These proteins are involved in regulating various cellular functions, including cytoskeletal structure, ion channel components, energy metabolism, and protein degradation. These findings indicated altered protein expressions in the pathogenesis of the early OA and illustrated a novel therapeutic avenue for the treatment of the early OA. Among the proteins examined, Pdia-3 in the knee joint has been reported to affect the integrity and function of osteoblast and chondrocyte cells [22-24].

Table 2. The proteins spot identification by MALDI-TOF/MS with Mascot search and NCBI BLAST database searching.

\begin{tabular}{|c|c|c|c|c|c|c|}
\hline $\begin{array}{l}\text { Spot } \\
\text { numb } \\
\text { er }\end{array}$ & $\begin{array}{l}\text { Name of } \\
\text { identified } \\
\text { protein }\end{array}$ & $\begin{array}{l}\text { Masc } \\
\text { ot } \\
\text { Score }\end{array}$ & $\begin{array}{l}\text { Entry } \\
\text { name }\end{array}$ & $\begin{array}{l}\text { BLAST } \\
\text { Score }\end{array}$ & $\begin{array}{l}\text { Molecul } \\
\text { ar } \\
\text { weight } \\
\text { [kDa] }\end{array}$ & $\begin{array}{l}\text { Theoretic } \\
\text { al PI } \\
\text { value }\end{array}$ \\
\hline P1324 & Beta-enolase & 74 & $\begin{array}{l}\text { ENOB_RA } \\
\mathrm{T}\end{array}$ & $\begin{array}{l}603.19763 \\
61\end{array}$ & 47.33743 & $\begin{array}{l}6.8865470 \\
57\end{array}$ \\
\hline P1325 & $\begin{array}{l}\text { Chloride } \\
\text { intracellular } \\
\text { channel protein } \\
1\end{array}$ & 96 & $\begin{array}{l}\text { CLIC1_RA } \\
\mathrm{T}\end{array}$ & $\begin{array}{l}260.73942 \\
64\end{array}$ & 27.30589 & $\begin{array}{l}4.9448124 \\
79\end{array}$ \\
\hline P1408 & $\begin{array}{l}\text { Malate } \\
\text { dehydrogenase }\end{array}$ & 35 & $\begin{array}{l}\text { MDHC_R } \\
\text { AT }\end{array}$ & 344.71 & 36.46005 & $\begin{array}{l}6.1681893 \\
25\end{array}$ \\
\hline P1409 & $\begin{array}{l}\text { Purine } \\
\text { nucleoside } \\
\text { phosphorylase }\end{array}$ & 136 & $\begin{array}{l}\text { PNPH_RA } \\
\mathrm{T}\end{array}$ & $\begin{array}{l}376.37444 \\
03\end{array}$ & 32.28104 & $\begin{array}{l}6.5191430 \\
97\end{array}$ \\
\hline P1412 & $\begin{array}{l}\text { Creatine kinase } \\
\text { M-type }\end{array}$ & 32 & $\begin{array}{l}\text { KCRM_R } \\
\text { AT }\end{array}$ & $\begin{array}{l}541.94220 \\
14\end{array}$ & 43.0178 & $\begin{array}{l}6.6320392 \\
61\end{array}$ \\
\hline P1413 & $\begin{array}{l}\text { L-lactate } \\
\text { dehydrogenase } \\
\text { A chain }\end{array}$ & 68 & $\begin{array}{l}\text { LDHA_RA } \\
\mathrm{T}\end{array}$ & $\begin{array}{l}589.40444 \\
03\end{array}$ & 36.42734 & $\begin{array}{l}9.2795628 \\
22\end{array}$ \\
\hline P1529 & $\begin{array}{l}\text { Protein } \\
\text { disulfide-isomer } \\
\text { ase A3 }\end{array}$ & 91 & $\begin{array}{l}\text { PDIA3_RA } \\
\mathrm{T}\end{array}$ & $\begin{array}{l}699.44954 \\
33\end{array}$ & 57.04387 & $\begin{array}{l}5.8294174 \\
23\end{array}$ \\
\hline P1981 & $\begin{array}{l}\text { Guanine } \\
\text { nucleotide-bindi } \\
\text { ng protein } \\
\text { subunit } \\
\text { beta-2-like }\end{array}$ & 50 & $\begin{array}{l}\text { GBLP_RA } \\
\mathrm{T}\end{array}$ & $\begin{array}{l}395.38840 \\
18\end{array}$ & 35.51073 & $\begin{array}{l}8.8766770 \\
99\end{array}$ \\
\hline
\end{tabular}


A

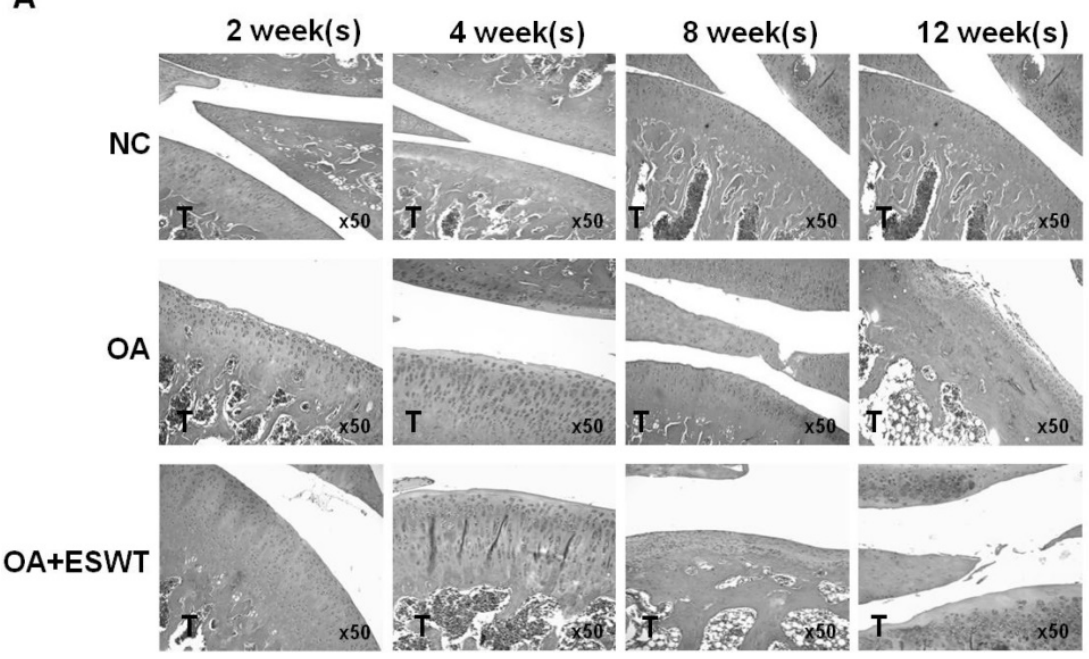

B

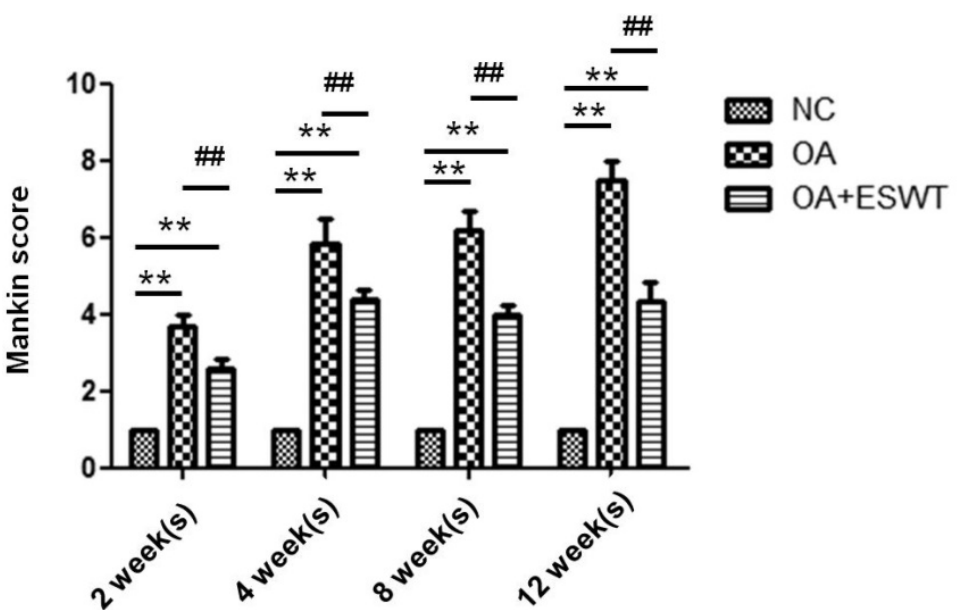

Figure 1. Histologic results of the early OA knee after ESWT. (A) Representative histological photographs of early OA knee after ESWT at 2, 4, 8, 12 week(s). Normal control (NC) showed normal structural integrity, cellularity, and cartilage height and tidemark integrity. T was indicated tibia. Specimens were stained by conventional hematoxylin-eosin. The total magnification is at $\times 50$. (B) Scores across a section of normal cartilage ranged from 0 to $1 . O A$ knee of section with clefts and modest proteoglycan depletion was assigned scores ranging from 4 to 8 . The OA+ESWT group showing regression of OA change was given scores from 2 to 5 . The **P $<0.001$ was OA and ESWT+OA compared with NC group and the \#\#P $<0.001$ was ESWT+OA group compared with OA group.

NC

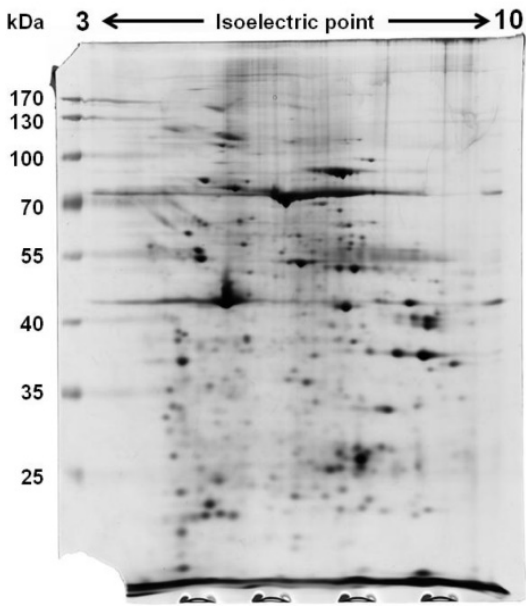

OA

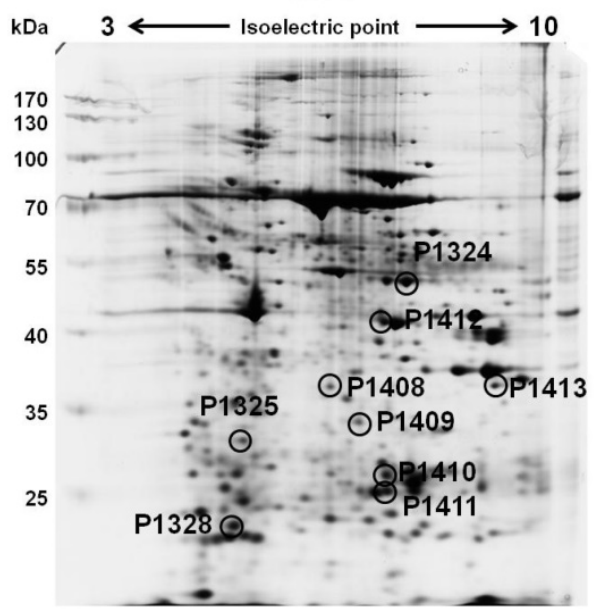

OA+ESWT

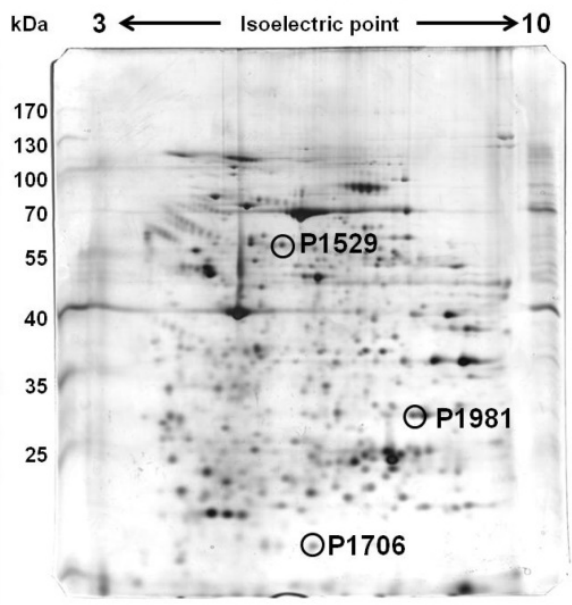

Figure 2. The articular cartilage and subchondral bone of left knee were analysis by two-dimensional gel electrophoresis from NC, OA and OA+ESWT groups. Protein spots of interest in two-dimensional gel electrophoretograms. ESWT induced or suppressed expression of several different proteins at 2 week(s). The level of significant spot was 1.5 fold increase or decrease. Twelve spots were found to be differentially abundant, including 9 spots decreased intensity and 3 spots increased signal observed in OA+ESWT group when compared with OA group at 2 week(s) after surgery. 


\section{Expressions of Pdia-3 and ERK1 after ESWT for early OA of the knee}

The spot of Pdia-3 exhibited a difference between the OA+ESWT group and the OA group at 2 week(s) after index surgery, first by de-staining and enzymatic in-gel digestion, followed by MALDI/TOF mass spectrometry analysis. The expression of Pdia-3 at each point in time is summarized in Figure 4. Significantly, up-regulation of the mRNA of Pdia-3 at each point in time was noted in the OA+ESWT group as compared with the OA group and the NC group, and it was particularly enhanced to a 13-fold increase at 2 week(s) higher than at 4, 8 and 12 week(s) after shockwave treatment in comparison with the OA group (Fig. 4A).

ERKs act as integration points for multiple biochemical signals, and are involved in osteoblast cellular processes such as proliferation, differentiation, transcription regulation and development $[12,25]$. Upon activation by Pdia-3 after ESWT, these kinases translocated to the nuclei of osteoblast cells, where they phosphorylated nuclear targets. Two alternatively-spliced transcript variants encoding different protein isoforms have been described for this gene. Significant increases the mRNA expression of ERK1 in the OA+ESWT group as compared with the $\mathrm{OA}$ and $\mathrm{NC}$ groups were observed, especially at 2 week(s) (Fig. 4B).

\section{Expressions of genes related to the rapid membrane signaling pathway after ESWT}

Application of shockwave therapy to the knee resulted in increases of bone formation markers, including OPG, ALP and MMP13. ALP increases when there is active bone formation, as it is a by-product of osteoblast activity [26, 27]. OPG in bone is the major determinant of bone mass and strength [28-30]. MMP13 is an established marker gene for bone formation [31, 32]. Significantly less pronounced subchondral bone remodeling with decreases in osteogenesis were noted in the OA group as compared with the OA+ESWT group. It appeared that application of ESWT to the medial tibia condyle of the knee improved osteogenesis and the bone turnover rate of the subchondral bone in knees affected by OA, and the results were comparable with those observed in the rats in the normal control group (Fig. 4C, 4D and $4 \mathrm{E})$.

\section{Effects of ESWT on Pdia-3 expression and the extracellular matrix in articular cartilage and subchondral bone of the early OA-affected knees}

We further investigated the expression of Pdia-3 by microscopic immunohistochemistry staining in the articular cartilage and subchondral bone of the knee at 2 week(s) (Fig. 5), and observed that the expression of Pdia- 3 was $40 \%$ and $15 \%$ more concentrated in the OA+ESWT group than in the OA group and NC group, respectively (Fig. 5A and 5B). The staining signal was particularly enriched in the superficial layer of the cartilage and the bone marrow of the subchondral bone, which indicated that Pdia-3 co-responded to ESWT in chondrocytes and the subchondral bone, as the expression of Pdia-3 was significantly increased in chondrocytes and bone in the OA+ESWT group as compared with the $\mathrm{OA}$ and $\mathrm{NC}$ groups.

The synthesis of arrows indicated the spot of interest between OA+ESWT and OA groups. ESWT promoted two proteins up-regulation including Pdia-3 (P1529) and guanine nucleotide-binding protein subunit beta-2-like (P1981). Moreover, six proteins were identified that shockwave knee suppressed, including Beta-enolase (P1324), chloride intracellular channel protein 1 (P1325), malate dehydrogenase (P1408), purine nucleoside phosphorylase (P1409), creatine kinase M-type (P1412) and L-lactate dehydrogenase A chain (P1413). Black arrow indicated the position of interest spots. an extracellular matrix of aggrecan and collagen type II was 
investigated in terms of its role in cartilage formation $[5,33,34]$. The OA+ESWT group showed significantly increased amounts of collagen type II (approximate 3 -fold increase) and aggrecan (approximate 20-fold increase) at 2 and 4 week(s) as compared with the NC and OA groups (Fig. 6A and 6B; Table 1).

\section{Discussion}

In this study, we expanded on prior research and confirmed that OA of the knee could be regressed by the application of shockwave therapy. Furthermore, we demonstrated a trend towards increased Pdia-3 biosynthetic activity in response to pulsed acoustic energy released by shockwave therapy. The mitogenic and anabolic activities of osteoblasts and chondrocytes increased relative to elevated ERK phosphorylation in the subchondral bone after ESWT. Growing evidence has indicated that Pdia-3-dependent mechanisms are involved in rapid responses to secosteroid 1, 25-dihydroxyvitamin D3 (1a, 25(OH)2D3) signaling in osteoblast and chondrocyte cells $[12,22,23]$. Pdia-3 has been identified as a potential alternative membrane-associated receptor for 1a,25(OH)2D31a,25(OH)2D3 directly regulates mineralization of osteoblasts and matrix formation of chondrocytes through the classic VDR-mediated genomic pathway and membrane receptor-mediated rapid responses via the Pdia-3-dependent pathway. Our study provided the first evidence that an effect of ESWT on OA of the knee is regulation of the protein Pdia-3, which is linked to the integrity and function of osteoblast and chondrocyte cells. This finding suggested that the proposed biomechanical pathway was likely to be conserved under shockwave therapy for the treatment of OA of the knee.

It is well-established that Pdia- 3 mediates the membrane response to $1 \mathrm{a}, 25(\mathrm{OH}) 2 \mathrm{D} 3$, including PLA2 stimulation-dependent rapid release of prostaglandin E2, activation of PKC, then regulates bone-related gene transcription and mineralization via phosphorylation of transcription factors such as ERK1/2 in osteoblast-like MC3T3-E1 cells [22]. Dr. Jiaxuan found that in Pdia-3-silenced (Sh-Pdia-3) cells, 1a,25(OH)2D3 failed to stimulate PKC and PGE2 reaction, and in Pdia-3-overexpressing cells (Ov-Pdia-3), the response to $1 \mathrm{a}, 25(\mathrm{OH}) 2 \mathrm{D} 3$ was augmented. These results suggested that Pdia- 3 could be a determining factor that correlates directly with the magnitude of the membrane response to $1 \mathrm{a}, 25(\mathrm{OH}) 2 \mathrm{D} 3$ [35]. The principal findings of this study showed that application of ESWT induced Pdia-3 up-regulation, leading to subchondral bone remolding after ACLT to create OA of the knee in rats. Gene expression analysis showed the ability of the cells to mineralize their extracellular matrix, and bone-related genes ALP, OPG and MMP13 significantly increased after shockwave therapy. The results were in agreement with prior studies that demonstrated that ESWT significantly enhances osteogenic factors reflecting local stimulation of bone formation during fracture-healing [36-38]. The results presented clearly showed that a number of genes encoding bone formation and related signaling molecules could potentially transduce osteogenic effects in response to shockwave treatment, changing the expression of Pdia-3 in the subchondral bone of the knee.

Pdia-3 mediated the signaling results in gene transcription, which can also modulate bone formation. The key component of ERK1 in this signaling pathway was also activated. ERK has been found to act as an important mediator for mechanical-stimulated proliferation and differentiation of osteogenic cells. Previous studies revealed that ERK is involved in shockwave-augmented bone formation in segmental defects within 14 days of treatment. The phosphorylation of ERK is active throughout the period of ESWT-induced bone regeneration and regulates the stimulation of biophysical shockwave therapy, triggering mitogenic and osteogenic responses in defects [39]. Our present data revealed that the signals of ERK were active, and it could play an important role in the signaling pathway of subchondral bone remolding 2 weeks after local application of shockwave therapy.

Several studies have reported positive effects of ESWT for OA of different joints in animals [40-44]. The exact mechanism of shockwave therapy remains unknown. Our current study provided the first evidence via immunohistochemical analysis that shockwave therapy can induce articular cartilage expression of Pdia-3, the critical transcription factor responsible for matrix formation of chondrocytes. Recent studies have indicated that $1 \mathrm{a}, 25(\mathrm{OH}) 2 \mathrm{D} 3$ rapidly stimulates membrane signaling via Pdia-3-dependent activation in growth-zone chondrocytes and promotes the production of matrix protein $[11,13,23,25,45,46]$. The present study showed a decrease in cartilage matrix loss and increased aggrecan and collagen II expressions in the shockwave treatment group, and explained the biomolecular mechanism of shockwave therapy in cartilage development and maintenance of the chondrocyte phenotype. Regression of OA of the knee was supported by the expression of Pdia-3 and biomarkers of the cartilage in the remolding surface of the articular area. 
A

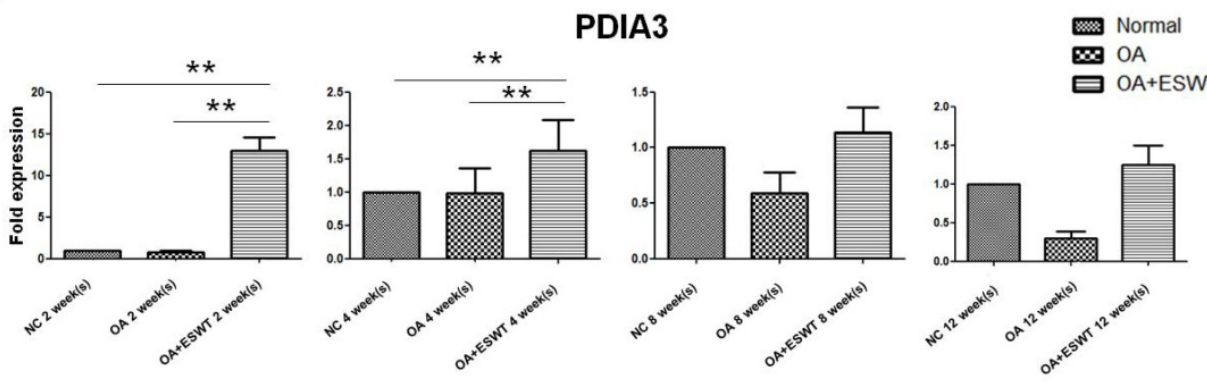

B

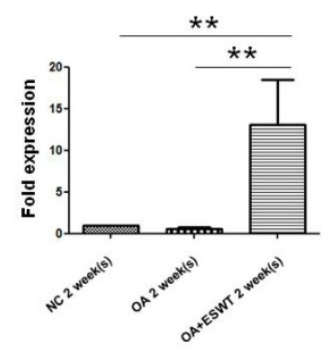

C

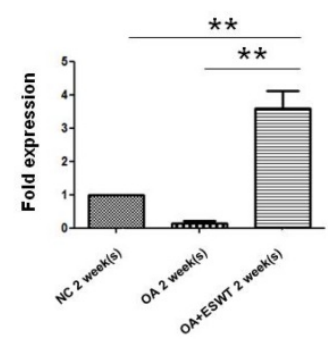

D

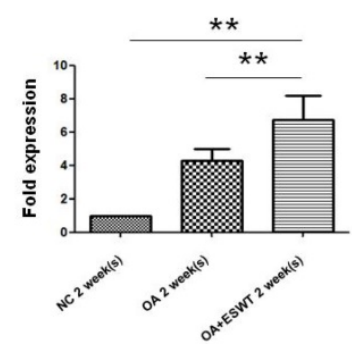

E

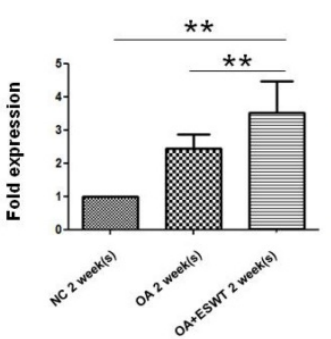

ERK1

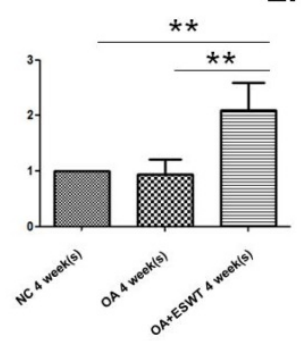

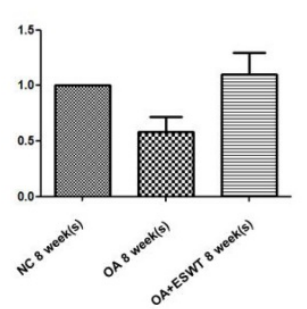

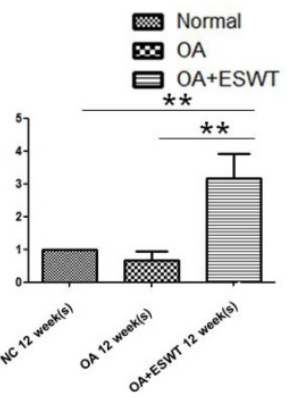

\section{Osteopontin}
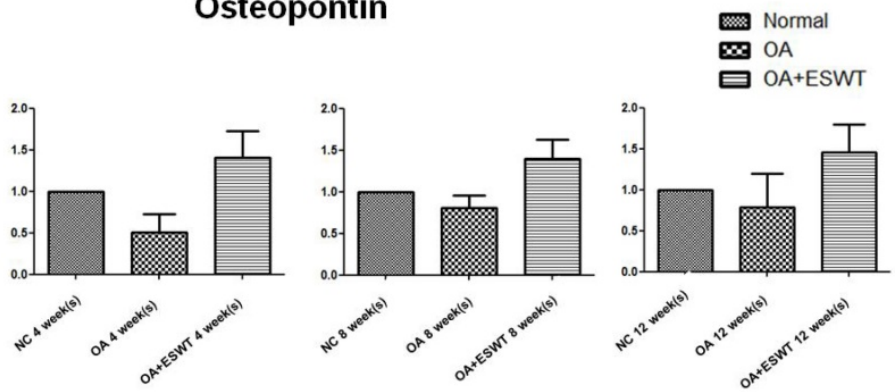

Alkaline phosphatase
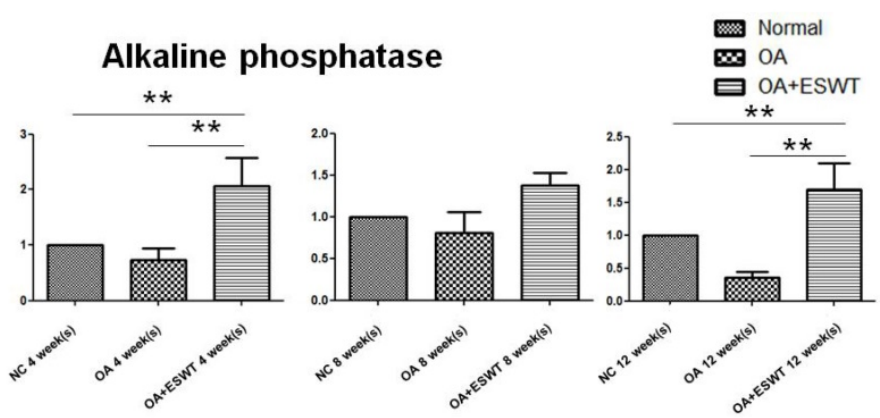

MMP13
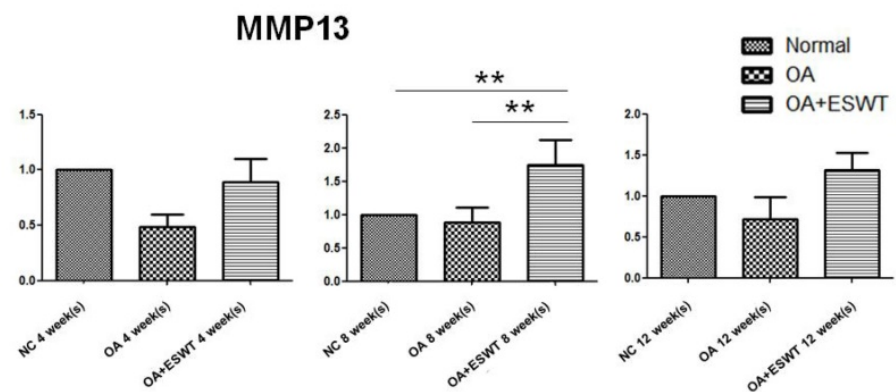

Figure 4. Effect of ESWT on Pdia-3 expression in early OA knee. (A) ESWT increased the expression of Pdia-3 at 2 week(s) and then decreased at 4, 8 and 12 week(s). When OA+ESWT compared with NC and OA groups, it had significant difference at 2 and 4 week(s) $(* *$ P $<0.001)$. (B) ESWT promoted ERK1 expression at 2 and 4 week(s) as compared with $N C$ and OA groups after index surgery (**P $<0.001$ ). (C-E) Effect of ESWT on bone formation markers in early OA knee. Shockwave therapy increased bone formation as implicated by active OPG, ALP and MMPI3 intensity, especially at 2 week(s) after treatment. (**P $<0.001)$ Real-time PCR was performed against 3 bone related genes: (C) osteoprotegerin; (D) alkaline phosphatase and (E) MMPI3. The OA+ESWT group showed significant more amount of bone turnover rate than as compared with $\mathrm{NC}$ and OA groups $(* * \mathrm{P}<0.001)$. 
A

A

NC

$O A$

OA+ESWT
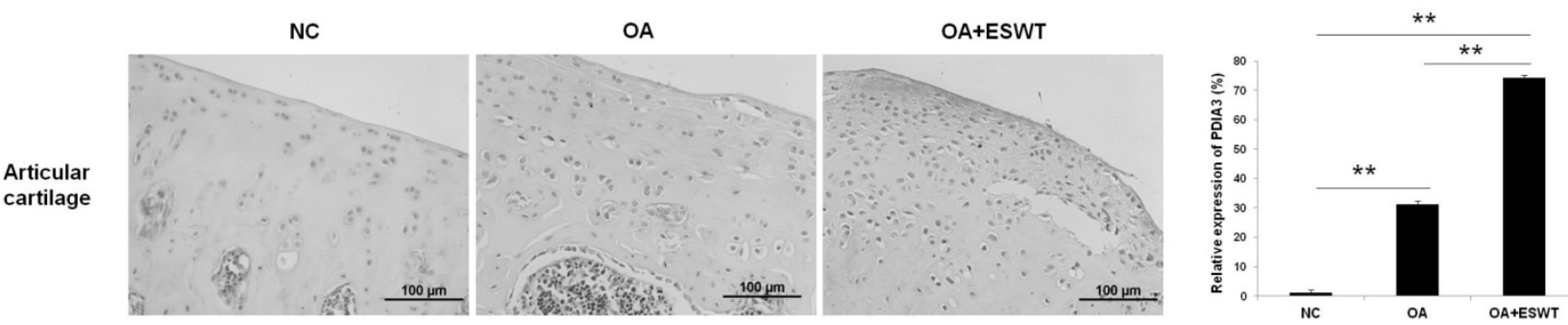

B

NC

$O A$

OA+ESWT
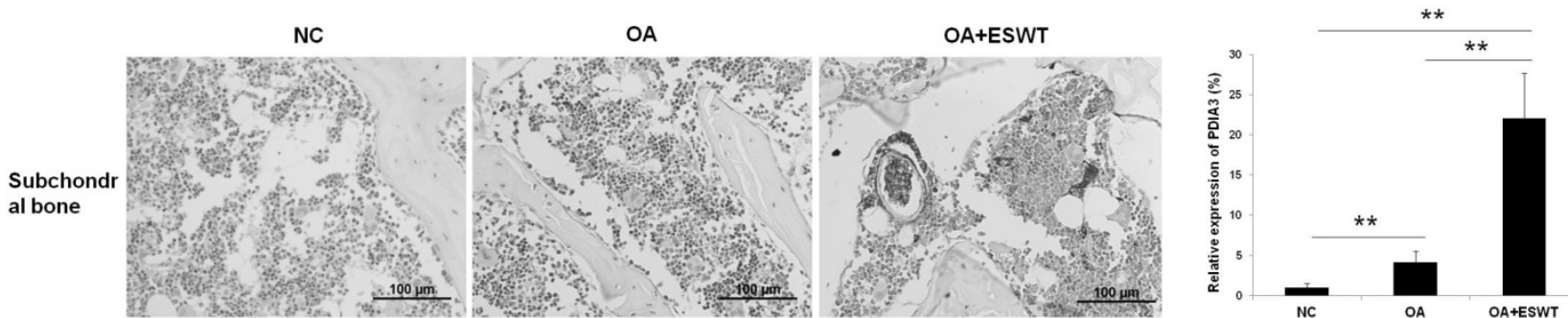

Figure 5. IHC staining of Pdia-3 in chondrocyte and subchondral bone with and without ESWT in early OA knee at 2 week(s). (A) The Pdia3 distributed over the cartilage and was enriched in the articular surface of OA+ESWT group. After measuring the staining signal, the OA+ESWT group showed significantly more expression of Pdia-3 in articular cartilage compared with OA knee.(**P $<0.001$ ). (B) The OA+ESWT group showed significantly expression of Pdia-3 in subchondral bone compared with NC and OA groups (**P $<0.001)$. The scar bar was $100 \mu \mathrm{m}$.

A

\section{Type II collagen}

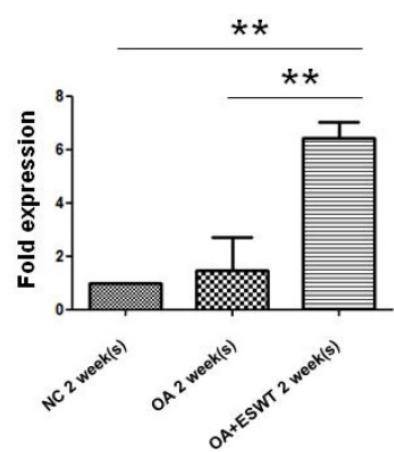

B

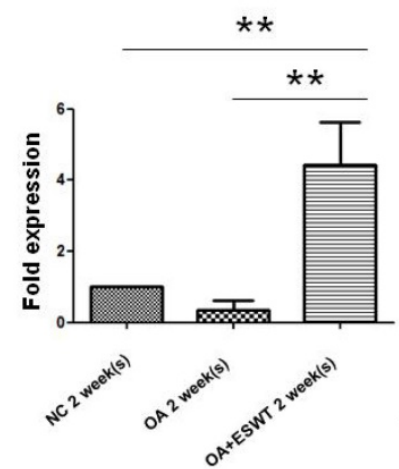

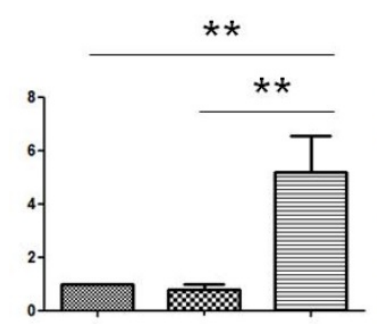
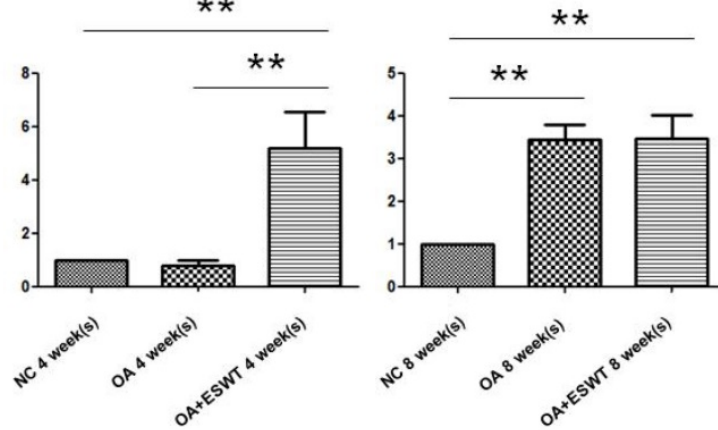

Aggrecan
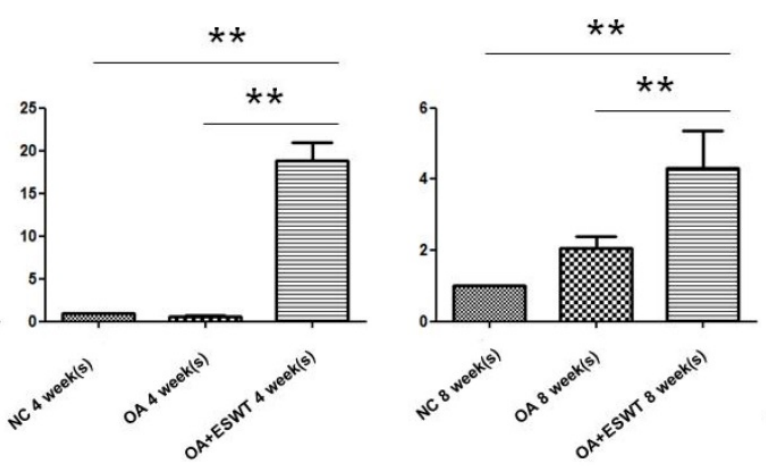
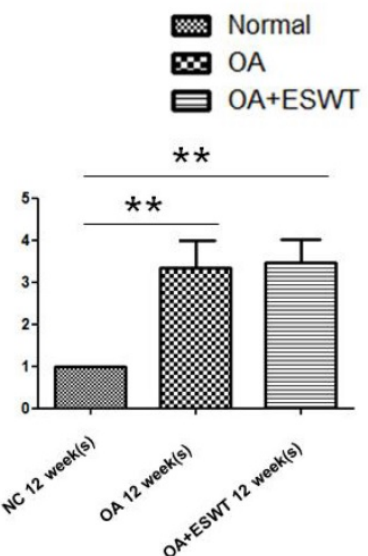

Figure 6. The effect of shockwave therapy induced expression of cartilage related gene in early OA knee. The OA+ESWT group showed significant increases in expression of collagen type II (A) and aggrecan (B) when compared with NC and OA groups at $2,4,8$ and 12 week(s) (**P < 0.001$)$. 
The effect of shockwave therapy in the osteoarthritic rat knee showed time-dependent chondroprotection [47]. We observed the most beneficial effects of shockwave therapy for OA of the knee after 2 weeks of shockwave application, and the effects of shockwave treatment appeared to continue until 12 weeks. These novel findings supported the concept that shockwave therapy provides a chondroprotective effect associated with improvement in subchondral bone remolding, a significant decrease in cartilage degradation, and an increase in chondrocyte activity in OA. Application of shockwave therapy to the subchondral bone was observed to be effective in a time-dependent fashion in $\mathrm{OA}$ of the knee.

The exact mechanism of ESWT remains unexplored. The innovative findings of this study may unveil new concepts of the biomolecular pathway and treatment of OA of the knee by ESWT. It appeared that local shockwave therapy application to the subchondral bone of the medial tibia condyle affected the entire knee joint through stimulation of osteoblast and chondrocyte cells via up-regulation of Pdia-3.

There were some limitations in this study. The data obtained from this study were based on small-animal experiments, and the results may differ in larger animals or human subjects. The dose conversion from small animals to larger animals or human subjects must be calculated following additional studies and clinical trials. The optimal ESWT dose and the ideal number of ESWT sessions remain unknown. Furthermore, different manufacturers use different indices of shockwave parameters, and the dose conversion formulae for the different devices are not readily available at the present time.

\section{Conclusions}

Our proteomic data revealed abundant significant Pdia-3 expression in joint tissue microenvironments that may have represented an active response to ESWT treatment, which potentially regulates the biological functions of chondrocytes and osteoblasts in OA of the knee. Furthermore, ESWT has potential benefits for the treatment of OA of the knee.

\section{Acknowledgements}

We are grateful to Kaohsiung Chang Gung Memorial Hospital for the supporting of this work. Funds are received support for the research study presented in this article. The funding sources are from Chang Gung Medical Foundation (No: CMRPG8B1311 and CMRPG8B1312).

\section{Conflicts of interest}

The authors declared that they did not receive any honoraria or consultancy fees in writing this manuscript. No benefits in any form have been received or will be received from a commercial party related directly or indirectly to the subject of this article. One author (Chin-Jen Wang) serves as a member of the advisory committee of Sanuwave, (Alpharetta, GA, USA) and this study is performed independent of the appointment. The remaining authors declared no conflict of interest.

\section{References}

1. Theis KA, Murphy L, Hootman JM, Helmick CG, Yelin E. Prevalence and correlates of arthritis-attributable work limitation in the US population among persons ages 18-64: 2002 National Health Interview Survey Data. Arthritis and rheumatism. 2007; 57: 355-63.

2. Kurz B, Lemke AK, Fay J, Pufe T, Grodzinsky AJ, Schunke M. Pathomechanisms of cartilage destruction by mechanical injury. Annals of anatomy = Anatomischer Anzeiger : official organ of the Anatomische Gesellschaft. 2005; 187: 473-85.

3. Min JL, Meulenbelt I, Kloppenburg M, van Duijn CM, Slagboom PE. Mutation analysis of candidate genes within the 2q33.3 linkage area for familial early-onset generalised osteoarthritis. European journal of human genetics : EJHG. 2007; 15: 791-9.

4. Haywood L, McWilliams DF, Pearson CI, Gill SE, Ganesan A, Wilson D, et al. Inflammation and angiogenesis in osteoarthritis. Arthritis and rheumatism. 2003; 48: 2173-7.

5. Bondeson J, Wainwright SD, Lauder S, Amos N, Hughes CE. The role of synovial macrophages and macrophage-produced cytokines in driving aggrecanases, matrix metalloproteinases, and other destructive and inflammatory responses in osteoarthritis. Arthritis research \& therapy. 2006; 8: R187.

6. Blom $\mathrm{AB}$, van Lent $\mathrm{PL}$, Libregts $\mathrm{S}$, Holthuysen $\mathrm{AE}$, van der Kraan PM, van Rooijen $\mathrm{N}$, et al. Crucial role of macrophages in matrix metalloproteinase-mediated cartilage destruction during experimental osteoarthritis: involvement of matrix metalloproteinase 3. Arthritis and rheumatism. 2007; 56: 147-57.

7. Yudoh K, Nguyen v T, Nakamura H, Hongo-Masuko K, Kato T, Nishioka K. Potential involvement of oxidative stress in cartilage senescence and development of osteoarthritis: oxidative stress induces chondrocyte telomere instability and downregulation of chondrocyte function. Arthritis research \& therapy. 2005; 7: R380-91.

8. Kim HA, Lee YJ, Seong SC, Choe KW, Song YW. Apoptotic chondrocyte death in human osteoarthritis. The Journal of rheumatology. 2000; 27: 455-62.

9. Hashimoto S, Setareh M, Ochs RL, Lotz M. Fas/Fas ligand expression and induction of apoptosis in chondrocytes. Arthritis and rheumatism. 1997; 40: 1749-55.

10. Johnson K, Svensson CI, Etten DV, Ghosh SS, Murphy AN, Powell HC, et al. Mediation of spontaneous knee osteoarthritis by progressive chondrocyte ATP depletion in Hartley guinea pigs. Arthritis and rheumatism. 2004; 50: 1216-25.

11. Sylvia VL, Del Toro F, Dean DD, Hardin RR, Schwartz Z, Boyan BD. Effects of 1alpha,25-(OH)(2) $\mathrm{D}(3)$ on rat growth zone chondrocytes are mediated via cyclooxygenase-1 and phospholipase A(2). Journal of cellular biochemistry Supplement. 2001; Suppl 36: 32-45.

12. Boyan BD, Chen J, Schwartz Z. Mechanism of Pdia3-dependent 1alpha,25-dihydroxy vitamin D3 signaling in musculoskeletal cells. Steroids. 2012; 77: 892-6.

13. Doroudi M, Boyan BD, Schwartz Z. Rapid 1alpha,25(OH)(2)D (3) membrane-mediated activation of $\mathrm{Ca}(2)(+) /$ calmodulin-dependent protein kinase II in growth plate chondrocytes requires Pdia3, PLAA and caveolae. Connective tissue research. 2014; 55 Suppl 1: 125-8.

14. Wang CJ, Ko JY, Kuo YR, Yang YJ. Molecular changes in diabetic foot ulcers. Diabetes research and clinical practice. 2011; 94: 105-10.

15. Wang CJ, Weng LH, Ko JY, Sun YC, Yang YJ, Wang FS. Extracorporeal shockwave therapy shows chondroprotective effects in osteoarthritic rat knee. Archives of orthopaedic and trauma surgery. 2011; 131: 1153-8.

16. Liu W, He J, Lin R, Liang J, Luo Q. Differential proteomics of the synovial membrane between bilateral and unilateral knee osteoarthritis in surgeryinduced rabbit models. Molecular medicine reports. 2016.

17. Liao W, Li Z, Zhang H, Li J, Wang K, Yang Y. Proteomic analysis of synovial fluid as an analytical tool to detect candidate biomarkers for knee osteoarthritis. International journal of clinical and experimental pathology. 2015; 8: 9975-89.

18. Ruiz-Romero C, Blanco FJ. The role of proteomics in osteoarthritis pathogenesis research. Current drug targets. 2009; 10: 543-56. 
19. Hayami T, Pickarski M, Zhuo $\mathrm{Y}$, Wesolowski GA, Rodan GA, Duong LT. Characterization of articular cartilage and subchondral bone changes in the rat anterior cruciate ligament transection and meniscectomized models of osteoarthritis. Bone. 2006; 38: 234-43.

20. Mankin HJ, Dorfman H, Lippiello L, Zarins A. Biochemical and metabolic abnormalities in articular cartilage from osteo-arthritic human hips. II. Correlation of morphology with biochemical and metabolic data. The Journal of bone and joint surgery American volume. 1971; 53: 523-37.

21. Yang MY, Chiang YC, Huang YT, Chen CC, Wang FS, Wang CJ, et al. Serum proteomic analysis of extracorporeal shock wave therapy-enhanced diabetic wound healing in a streptozotocin-induced diabetes model. Plast Reconstr Surg. 2014; 133: 59-68.

22. Chen J, Doroudi M, Cheung J, Grozier AL, Schwartz Z, Boyan BD. Plasma membrane Pdia3 and VDR interact to elicit rapid responses to 1alpha,25(OH)(2)D(3). Cellular signalling. 2013; 25: 2362-73.

23. Doroudi M, Olivares-Navarrete R, Boyan BD, Schwartz Z. A review of 1alpha,25(OH)2D3 dependent Pdia3 receptor complex components in Wnt5a non-canonical pathway signaling. The Journal of steroid biochemistry and molecular biology. 2015; 152: 84-8.

24. Doroudi M, Olivares-Navarrete R, Hyzy SL, Boyan BD, Schwartz Z. Signaling components of the 1alpha,25(OH)2D3-dependent Pdia3 receptor complex are required for Wnt5a calcium-dependent signaling. Biochimica et biophysica acta. 2014; 1843: 2365-75.

25. Doroudi M, Schwartz Z, Boyan BD. Phospholipase A2 activating protein is required for 1alpha,25-dihydroxyvitamin D3 dependent rapid activation of protein kinase $\mathrm{C}$ via Pdia3. The Journal of steroid biochemistry and molecular biology. 2012; 132: 48-56.

26. Avbersek-Luznik I, Gmeiner Stopar T, Marc J. Activity or mass concentration of bone-specific alkaline phosphatase as a marker of bone formation. Clinical chemistry and laboratory medicine. 2007; 45: 1014-8.

27. van Straalen JP Sanders E Prummel MF Sanders GT, Bone-alkaline phosphatase as indicator of bone formation. Clinica chimica acta; international journal of clinical chemistry. 1991; 201: 27-33

28. Tanaka $\mathrm{H}$, Mine $\mathrm{T}$, Ogasa $\mathrm{H}$, Taguchi $\mathrm{T}$, Liang CT. Expression of RANKL/OPG during bone remodeling in vivo. Biochemical and biophysical research communications. 2011; 411: 690-4.

29. Simonet WS, Lacey DL, Dunstan CR, Kelley M, Chang MS, Luthy R, et al. Osteoprotegerin: a novel secreted protein involved in the regulation of bone density. Cell. 1997; 89: 309-19.

30. Udagawa N, Takahashi N, Yasuda H, Mizuno A, Itoh K, Ueno Y, et al. Osteoprotegerin produced by osteoblasts is an important regulator in osteoclast development and function. Endocrinology. 2000; 141: 3478-84.

31. Tuckermann JP, Pittois K, Partridge NC, Merregaert J, Angel P. Collagenase-3 (MMP-13) and integral membrane protein 2a (Itm2a) are marker genes of chondrogenic/osteoblastic cells in bone formation: sequential temporal, and spatial expression of Itm2a, alkaline phosphatase, MMP-13, and osteocalcin in the mouse. Journal of bone and mineral research : the official journal of the American Society for Bone and Mineral Research. 2000; 15: 1257-65.

32. Hayami T, Kapila YL, Kapila S. MMP-1 (collagenase-1) and MMP-13 (collagenase-3) differentially regulate markers of osteoblastic differentiation in osteogenic cells. Matrix biology : journal of the International Society for Matrix Biology. 2008; 27: 682-92.

33. Dufield DR, Nemirovskiy OV, Jennings MG, Tortorella MD, Malfait AM, Mathews WR. An immunoaffinity liquid chromatography-tandem mass spectrometry assay for detection of endogenous aggrecan fragments in biological fluids: Use as a biomarker for aggrecanase activity and cartilage degradation. Analytical biochemistry. 2010; 406: 113-23.

34. Fraser A, Fearon U, Billinghurst RC, Ionescu M, Reece R, Barwick T, et al. Turnover of type II collagen and aggrecan in cartilage matrix at the onset of inflammatory arthritis in humans: relationship to mediators of systemic and local inflammation. Arthritis and rheumatism. 2003; 48: 3085-95.

35. Chen J, Lobachev KS, Grindel BJ, Farach-Carson MC, Hyzy SL, El-Baradie KB, et al. Chaperone properties of pdia3 participate in rapid membrane actions of 1alpha,25-dihydroxyvitamin d3. Molecular endocrinology. 2013; 27: 1065-77.

36. Kaspar D, Neidlinger-Wilke C, Holbein O, Claes L, Ignatius A. Mitogens are increased in the systemic circulation during bone callus healing. Journal of orthopaedic research : official publication of the Orthopaedic Research Society. 2003; $21: 320-5$.

37. Taniguchi T, Matsumoto T, Shindo H. Changes of serum levels of osteocalcin, alkaline phosphatase, IGF-I and IGF-binding protein-3 during fracture healing. Injury. 2003; 34: 477-9.

38. Wang FS, Wang CJ, Sheen-Chen SM, Kuo YR, Chen RF, Yang KD. Superoxide mediates shock wave induction of ERK-dependent osteogenic transcription factor (CBFA1) and mesenchymal cell differentiation toward osteoprogenitors. The Journal of biological chemistry. 2002; 277: 10931-7.

39. Chen YJ, Kuo YR, Yang KD, Wang CJ, Sheen Chen SM, Huang HC, et al. Activation of extracellular signal-regulated kinase (ERK) and p38 kinase in shock wave-promoted bone formation of segmental defect in rats. Bone. 2004; 34: 466-77.

40. Dahlberg J, Fitch G, Evans RB, McClure SR, Conzemius M. The evaluation of extracorporeal shockwave therapy in naturally occurring osteoarthritis of the stifle joint in dogs. Veterinary and comparative orthopaedics and traumatology : VCOT. 2005; 18: 147-52.

41. Frisbie DD, Kawcak CE, Mcllwraith CW. Evaluation of the effect of extracorporeal shock wave treatment on experimentally induced osteoarthritis in middle carpal joints of horses. American journal of veterinary research. 2009; 70: 449-54

42. Mueller M, Bockstahler B, Skalicky M, Mlacnik E, Lorinson D. Effects of radial shockwave therapy on the limb function of dogs with hip osteoarthritis. The Veterinary record. 2007; 160: 762-5.

43. Ochiai N, Ohtori S, Sasho T, Nakagawa K, Takahashi K, Takahashi N, et al. Extracorporeal shock wave therapy improves motor dysfunction and pain originating from knee osteoarthritis in rats. Osteoarthritis and cartilage / OARS, Osteoarthritis Research Society. 2007; 15: 1093-6.

44. Revenaugh MS. Extracorporeal shock wave therapy for treatment of osteoarthritis in the horse: clinical applications. The Veterinary clinics of North America Equine practice. 2005; 21: 609-25, vi.

45. Schwartz Z, Graham EJ, Wang L, Lossdorfer S, Gay I, Johnson-Pais TL, et al. Phospholipase A2 activating protein (PLAA) is required for 1alpha,25(OH)2D3 signaling in growth plate chondrocytes. Journal of cellular physiology. 2005; 203: 54-70.

46. Boyan BD, Wong KL, Wang L, Yao H, Guldberg RE, Drab M, et al. Regulation of growth plate chondrocytes by 1,25-dihydroxyvitamin $\mathrm{D} 3$ requires caveolae and caveolin-1. Journal of bone and mineral research : the official journal of the American Society for Bone and Mineral Research. 2006; 21: 1637-47.

47. Wang CJ, Sun YC, Wong T, Hsu SL, Chou WY, Chang HW. Extracorporeal shockwave therapy shows time-dependent chondroprotective effects in osteoarthritis of the knee in rats. The Journal of surgical research. 2012; 178: $196-205$. 